REPRESENTATION THEORY

An Electronic Journal of the American Mathematical Society

Volume 12, Pages 208-224 (April 17, 2008)

S 1088-4165(08)00328-2

\title{
WEIGHTED PROJECTIVE SPACES AND MINIMAL NILPOTENT ORBITS
}

\author{
CARLO A. ROSSI
}

\begin{abstract}
We investigate (twisted) rings of differential operators on the resolution of singularities of an irreducible component $\bar{X}$ of $\bar{O}_{\min } \cap \mathfrak{n}_{+}$(where $\bar{O}_{\text {min }}$ is the (Zariski) closure of the minimal nilpotent orbit of $\mathfrak{s p}_{2 n}$ and $\mathfrak{n}_{+}$ is the Borel subalgebra of $\mathfrak{s p}_{2 n}$ ) using toric geometry, and show that they are homomorphic images of a certain family of associative subalgebras of $U\left(\mathfrak{s p}_{2 n}\right)$, which contains the maximal parabolic subalgebra $\mathfrak{p}$ determining $\bar{O}_{\min }$. Further, using Fourier transforms on Weyl algebras, we show that (twisted) rings of well-suited weighted projective spaces are obtained from the same family of subalgebras. Finally, we investigate this family of subalgebras from the representation-theoretical point of view and, among other things, rediscover in a different framework irreducible highest weight modules for the UEA of $\mathfrak{s p}_{2 n}$.
\end{abstract}

\section{INTRODUCTION}

Let us consider the Lie algebra $\mathfrak{g}=\mathfrak{s p}_{2 n}$ : the ring $\mathcal{D}(\bar{X})$ of differential operators on $\bar{X}$, an irreducible component of $\bar{O}_{\min } \cap \mathfrak{n}_{+}, \bar{O}_{\text {min }}$ being the Zariski closure of the minimal nilpotent orbit of $\mathfrak{g}$ and $\mathfrak{n}_{+}$the Borel subalgebra of $\mathfrak{g}$, has been discussed in detail in [6] and independently in [7; for an explicit characterization of $\bar{X}$, we refer to 6] and Section 2. The variety $\bar{X}$ has the property that it admits a surjective morphism

$$
U(\mathfrak{g}) \rightarrow \mathcal{D}(\bar{X})
$$

while Levasseur et al. [6] have obtained (11) as a special case in a more general framework, invoking Fourier transform, Musson 7] highlighted the fact that $\bar{X}$ is a toric variety (affine and singular). In particular, the toric structure permits 8 to find an explicit realization of the epimorphism (1) by constructing generators and relations of $\mathcal{D}(\bar{X})$ using homogeneous coordinates on $\bar{X}$. The construction of such coordinates for $\bar{X}$ has the advantage of producing almost without effort an explicit resolution of singularities of $\bar{X}$, which we denote by $\widetilde{X}$; explicitly, $\widetilde{X}$ can be identified with the Serre bundle $\mathcal{O}(-2)$ over $\mathbb{P}^{n-1}$. The toric structure of $\widetilde{X}$ allows 8 to compute generators and relations for any twisted ring $\mathcal{D}_{\mathcal{O}\left(\ell D_{0}\right)}(\widetilde{X}), D_{0}$ being a generator of the Picard group of $\widetilde{X}$ and $\ell$ an integer.

Our main result is a version of (1) for $\widetilde{X}$, for which we need some preparations. Observe that the construction of $\bar{X}$ requires the choice of a maximal parabolic subalgebra $\mathfrak{p}$ of $\mathfrak{g}$. In the case at hand, $\mathfrak{p}$ splits as $\mathfrak{m} \oplus \mathfrak{r}_{+}$, where $\mathfrak{m}$ is a reductive algebra, with one-dimensional center $\mathfrak{z}$ and semisimple part $\mathfrak{s l}_{n}$, while $\mathfrak{r}_{+}$is the nilradical,

Received by the editors August 17, 2007 and, in revised form, November 8, 2007.

2000 Mathematics Subject Classification. Primary 13N10.

(c)2008 American Mathematical Society Reverts to public domain 28 years from publication 
which, as an $\mathfrak{m}$-module, is isomorphic to the finite-dimensional, irreducible module $L\left(2 \varpi_{1}\right), \varpi_{i}, i=1, \ldots, n-1$ being the $i$-th fundamental weight of $\mathfrak{s l}_{n}$. We have the triangular decomposition $\mathfrak{g}=\mathfrak{r}_{-} \oplus \mathfrak{m} \oplus \mathfrak{r}_{+}$, where $\mathfrak{r}_{-}$is, as an $\mathfrak{m}$-module, isomorphic to $L\left(2 \varpi_{n-1}\right), \varpi_{i}$ being the fundamental weights of $\mathfrak{s l}_{n}$.

Theorem 1.1. For any integer $\ell$, consider the subalgebra $\mathfrak{A}_{\ell}$ of $U(\mathfrak{g})$, generated by $1, \mathfrak{m}, \mathfrak{r}_{-}$and $\mathfrak{r}_{+} \mathfrak{z} \ell$; then, for any integer number $\ell$, there is a surjective morphism

$$
\mathfrak{A}_{\ell} \rightarrow \mathcal{D}_{\mathcal{O}\left(\ell D_{0}\right)}(\widetilde{X}) \text {. }
$$

Here, $\mathfrak{z}_{\ell}$ denotes the 1 -dimensional subspace of $U(\mathfrak{g})$ spanned by $z_{\ell}:=z+\frac{\ell}{2}+\frac{n}{4}$, where $z$ is the generator of $\mathfrak{z}$ chosen accordingly to Subsection 3.2, Remark (vii) of [6].

In particular, we will show that there is a surjective morphism from $U(\mathfrak{p})$ to the subalgebra of $D_{\mathcal{O}\left(\ell D_{0}\right)}(\widetilde{X})$ of differential operators of non-positive degree, for any integer $\ell$, and a surjective morphism from $U\left(\mathfrak{s l}_{n}\right)$ to the subalgebra of differential operators of degree 0 ; observe that there is a natural right $S L_{n}$-action on $\widetilde{X}$, through which one obtains the vector fields inducing the differential operators in $U\left(\mathfrak{s l}_{n}\right)$. The notion of degree needs a brief explanation: all rings of differential operators considered here are subrings of the Weyl algebra $\mathcal{D}\left(\mathbb{A}^{n+1}\right)$, hence all elements are linear combinations of differential monomials $Q^{\mu} P^{\nu}, Q_{i}$, resp. $P_{i}, i=1, \ldots, n+1$, being multiplication operators by the variables $Q_{i}$, resp. differential operators w.r.t. $Q_{i}$, and $\mu, \nu$ are multi-indices with $n+1$ components. The degree of such a monomial is simply $\sum_{i=1}^{n+1} \tau_{i}$, where $\tau=\mu-\nu$.

As shown in [3], there exists an explicit isomorphism between (twisted) rings of differential operators on projective $n$-space $\mathbb{P}^{n}$ and on the blow-up $\widetilde{\mathbb{A}^{n}}$ of the affine $n$-space $\mathbb{A}^{n}$; analogously, there is an isomorphism between (twisted) rings of differential operators on the weighted projective $n$-space $Y=\mathbb{P}^{n}(1, \ldots, 1,2)$ and on the resolution $\widetilde{X}$.

Theorem 1.2. For any even integer $\ell$, there is a ring isomorphism

$$
\mathcal{D}_{\mathcal{O}\left(\ell D_{0}\right)}(\tilde{X}) \rightarrow \mathcal{D}_{\mathcal{O}(\ell-2)}(Y) \text {. }
$$

The isomorphism in the previous theorem needs some explanation. It is realized concretely by means of Fourier transform [9], using the main result of [3]: we simply observe that the weighted projective space $Y$ and $\widetilde{X}$ are related to each other by means of $I$-reflections. We notice that this phenomenon cannot be observed on $\bar{X}$. Differently from the usual projective space, the weighted projective space $Y$ is a singular toric variety; hence, the Main Theorem of [3] cannot be readily applied. Weighted projective spaces share, on the other hand, many of the properties of usual projective spaces; in particular, they possess natural Serre sheaves $\mathcal{O}(m)$, $m$ being an integer, which are invertible if and only if $m$ is even. Recalling the ingredients of the Main Theorem of [3], we have to determine for which choices of an integer $\ell$ the image of the invertible sheaf $\mathcal{O}\left(\ell D_{0}\right)$ on $\widetilde{X}$ in $A_{n-1}(Y)$ corresponds to an invertible sheaf on $Y$; for the same reasons as above, only even integers $\ell$ will do the job.

We may further combine both theorems to get a useful corollary.

Corollary 1.3. For any even integer $\ell$, there is a surjective morphism

$$
\mathfrak{A}_{\ell+2} \rightarrow \mathcal{D}_{\mathcal{O}(\ell)}(Y) \text {. }
$$


As a consequence, there is a surjective morphism from $U(\mathfrak{p})$ to the subalgebra $D_{\mathcal{O}(\ell)}(Y)$ of differential operators of non-positive degree; observe also that there is an $M$-action on $Y$, with $M$ as above. We notice that, unlike in the case of usual projective spaces, one cannot realize weighted projective spaces as $S p_{2 n^{-}}$ homogeneous spaces.

Finally, the twisted $\operatorname{ring} \mathcal{D}_{\mathcal{O}\left(\ell D_{0}\right)}(\tilde{X})$, resp. $\mathcal{D}_{\mathcal{O}(\ell)}(Y)$, where $\ell$ is an integer, resp. an even integer, acts on the cohomology groups of $\widetilde{X}$, resp. $Y$, with values in the invertible sheaf $\mathcal{O}\left(\ell D_{0}\right)$, resp. $\mathcal{O}(\ell)$. The cohomology groups can be explicitly computed in both cases (see Subsections 2.2 and 4.2), and via the previous theorems, are endowed with the structure of modules over $\mathfrak{A}_{\ell}$ and $\mathfrak{A}_{\ell+2}$, respectively. The representation theory of algebras of the form $\mathfrak{A}_{\ell}$ presents certain similar features to the representation theory of $\mathfrak{g}$; in particular, we may introduce a category of $\mathfrak{A}_{\ell^{-}}$ modules, which plays the rôle of the Bernstein-Gel'fand-Gel'fand (BGG) category $\mathcal{O}$ of $\mathfrak{g}$. We examine this category and find a criterion expressing the possibility for such an $\mathfrak{A}_{\ell}$-module to lift to a $\mathfrak{g}$-module. Of particular importance is the following result, which generalizes results of Levasseur et al. 6] regarding the $\mathfrak{g}$-module of regular functions on $\bar{X}$.

Theorem 1.4. For any non-positive integer $\ell$, the space of global sections of the invertible sheaf $\mathcal{O}\left(\ell D_{0}\right)$ over $\tilde{X}$ is isomorphic to $L\left(-\frac{1}{2} \varpi_{n}\right)$, if $\ell$ is even, or to $L\left(\varpi_{n-1}-\frac{3}{2} \varpi_{n}\right)$, if $\ell$ is odd.

Here, $L(\mu)$, for some weight $\mu$ of $\mathfrak{h}$, the Cartan subalgebra of $\mathfrak{g}$, denotes the unique irreducible quotient of the Verma module of highest weight $\mu ; \varpi_{i}, i=1, \ldots, n$, denotes the $i$-th fundamental weight of $\mathfrak{g}$. Observe that in both cases, the highest weights belong to the same Weyl orbit of weights of $\mathfrak{h}$.

In all other cases, we are able to characterize explicitly the cohomology groups as irreducible highest weight $\mathfrak{A}_{\ell^{-}}$or $\mathfrak{A}_{\ell+2}$-modules in the aforementioned category; in particular, we are able to decompose them into irreducible, finite-dimensional $\mathfrak{s l}_{n}$-modules (recall the observations about the $M$-action on both varieties $\widetilde{X}$ and $Y$ ), with a grading induced by $\mathfrak{z} \ell$ or $\mathfrak{z} \ell+2$ and by the modules $\mathfrak{r}_{+}$and $\mathfrak{r}_{-}$; see Subsubsections 5.2 .1 and 5.2 .2 for more details on cohomology groups as $\mathfrak{A}_{\ell}$-modules.

\section{A SUItABle iRREDUCIBLE COMPONENT OF $\bar{O}_{\min } \cap \mathfrak{n}_{+} \operatorname{IN} \mathfrak{s p}_{2 n}$}

We consider the root system of $\mathfrak{g}=\mathfrak{s p}_{2 n}$ following the notations of Bourbaki [2]: the only simple root of $\mathfrak{g}$ whose coefficient in any root decomposition is at most 1 is $\alpha_{n}$, hence $\alpha_{n}$ specifies a unique maximal parabolic subalgebra $\mathfrak{p}$ with decomposition into reductive part $\mathfrak{m}$ and nilradical $\mathfrak{r}_{+}$containing the highest root $e_{\beta}$. The irreducible component $\bar{X}$ of $\bar{O}_{\min } \cap \mathfrak{n}_{+}$is, by definition, the Zariski closure of the adjoint cone of highest weight $X=M e_{\beta}, M$ a connected, simply connected algebraic group with Lie algebra $\mathfrak{m}$; it corresponds to the variety of quadratic forms on $\mathbb{C}^{n}$ of rank less or equal than 1 ; see e.g. Section 3.2 of [6]. This characterization of $\bar{X}$ makes evident its toric structure, the corresponding fan $\Delta$ consists of a single cone in $N \cong \mathbb{Z}^{n}$ spanned by $n$ vectors $k_{i}, i=1, \ldots, n$, such that (i) they can be completed to $n+1$ vectors generating $N$, and (ii) they satisfy $\sum_{i=1}^{n} k_{i}=2 k_{n+1}$. Observe that $\Delta$ is not regular in the sense of [3] and $\bar{X}$ is singular.

For subsequent computations, we need to characterize the toric variety $\bar{X}$ as a quotient of a quasi-affine variety $Y$ by some torus, in the same spirit of [7]. By 
the previous characterization of the fan $\Delta$, the quasi-affine variety is simply the product $\mathbb{A}^{n} \times \mathbb{A}^{1} \backslash\{0\}$, while the torus action is

$$
\begin{aligned}
\mathbb{C}^{\times} \times \mathbb{A}^{n} \times \mathbb{A}^{1} \backslash\{0\} & \ni\left(u, Q_{1}, \ldots, Q_{n+1}\right) \\
& \mapsto\left(u Q_{1}, \ldots, u Q_{n}, u^{-2} Q_{n+1}\right) \in \mathbb{A}^{n} \times \mathbb{A}^{1} \backslash\{0\} .
\end{aligned}
$$

Remark 2.1. The variety $\bar{X}$ is a special case $\left(\mathfrak{g}\right.$ of type $C_{n}$ ) of many (affine and mostly singular) varieties appearing in [6], for a semisimple Lie algebra $\mathfrak{g}$ of type $A_{n}, B_{n}, C_{n}, D_{n}, E_{6}$ and $E_{7}$; such varieties have the remarkable property that they admit a surjective homomorphism from $U(\mathfrak{g})$ onto their rings of regular differential operators. In [6], the last statement is proved in general by means of Fourier transform; in [3], in the particular case $\mathfrak{g}$ of type $A_{n}$, the statement is alternatively proved using partial Fourier transforms on toric varieties and classical results of [1]; finally, if $\mathfrak{g}$ is of type $C_{n}$, it is also proved in [7] using toric geometry and an explicit characterization of the ring of differential operators; see also Subsection 2.3 for more details.

2.1. Resolution of singularities. We consider the fan $\widetilde{\Delta}$ obtained from $\Delta$, whose one-dimensional rays are spanned by the vectors $k_{i}, i=1, \ldots, n+1$, and whose cones of maximal dimension are spanned by exactly $n$ generating vectors out of the $k_{i}$, except the cone spanned by $k_{1}, \ldots, k_{n}: \widetilde{\Delta}$ is now regular in the sense of 3 ] and determines a toric variety $\widetilde{X}$, which is, by direct construction, a resolution of singularities of $\bar{X}$. Concretely, using homogeneous coordinates, $\widetilde{X}$ is the quotient $\left(\mathbb{A}^{n} \backslash\{0\} \times \mathbb{A}^{1}\right) / / \mathbb{C}^{\times}$with torus action

$$
\begin{aligned}
\mathbb{C}^{\times} \times \mathbb{A}^{n} \backslash\{0\} \times \mathbb{A}^{1} & \ni\left(u, Q_{1}, \ldots, Q_{n+1}\right) \\
& \mapsto\left(u Q_{1}, \ldots, u Q_{n}, u^{-2} Q_{n+1}\right) \in \mathbb{A}^{n} \backslash\{0\} \times \mathbb{A}^{1} .
\end{aligned}
$$

The variety $\tilde{X}$ is covered by $n$ open affine subsets, each isomorphic to the affine space $\mathbb{A}^{n}$; observe that $\tilde{X}$ is identified with the line bundle $\mathcal{O}(-2)$ over $\mathbb{P}^{n-1}$. We notice that the algebraic group $G L_{n}$ acts from the right on $\widetilde{X}$ by inverting the natural $G L_{n}$-action in the first $n$ homogeneous coordinates of $\widetilde{X}$.

2.2. The cohomology of $\widetilde{X}$. Since $\widetilde{\Delta}$ is regular, the Picard group of $\widetilde{X}$ is isomorphic to the group $A_{n-1}(\widetilde{X})$ of Weil divisors modulo linear equivalence. More explicitly, the Picard group is spanned by the $T$-divisors $D_{i}$ (see [4]) associated to the 1-dimensional cones of $\widetilde{\Delta}$, with the additional relations $D_{i} \sim D_{j}, i, j \in\{1, \ldots, n\}$, and $2 D_{i} \sim-D_{n+1}, i \in\{1, \ldots, n\}$; thus $A_{n-1}(\widetilde{X})$ is spanned by, say, $D_{0}:=D_{1}$ over $\mathbb{Z}$. For any Cartier divisor $\ell D_{0}$, where $\ell$ is an integer, the corresponding invertible sheaf $\mathcal{O}\left(\ell D_{0}\right)$ is a sheaf of covariants (see 8$]$ ) associated to the character $u \mapsto u^{\ell}$ of $\mathbb{C}^{\times}$. Further, the identification $\mathcal{O}\left(\ell D_{0}\right) \cong \mathcal{O}(\ell)$ on the divisor $D_{n+1} \cong \mathbb{P}^{n-1}$ holds true (one may thus view $\widetilde{X}$ as a "weighted" version of the blow-up $\widetilde{\mathbb{A}^{n}}$ ).

Theorem 2.2. For any integer $\ell$, there is an isomorphism

$$
H^{\bullet}\left(\tilde{X}, \mathcal{O}\left(\ell D_{0}\right)\right) \cong \bigoplus_{m \geq 0} H^{\bullet}\left(\mathbb{P}^{n-1}, \mathcal{O}(\ell+2 m)\right)
$$

Proof. The proof follows along the same lines of the proof of Theorem 4.7 in $[3$, the only difference being that the fiber coordinate on $\widetilde{X}$, viewed as a bundle over $\mathbb{P}^{n-1}$, has weight -2 , which produces the factor 2 in the shift on the right-hand side of the previous isomorphism. 
As a consequence, the cohomology of $\widetilde{X}$ with values in the invertible sheaf $\mathcal{O}\left(\ell D_{0}\right)$ is non-trivial exactly in degree 0 and $n-1$; furthermore, the 0 -th cohomology is always non-trivial and infinite-dimensional, while the $n-1$-th cohomology is finitedimensional and non-trivial exactly when $\ell \leq-n$.

Recalling the cohomology of projective space $\mathbb{P}^{n-1}$, we know that the 0 -th cohomology of $\mathbb{P}^{n-1}$ with values in $\mathcal{O}(m)$ is spanned by monomials $Q_{1}^{\mu_{1}} \cdots Q_{n}^{\mu_{n}}$ with $\mu_{i} \geq 0$ and $\sum_{i=1}^{n} \mu_{i}=m, m \geq 0$, and that the $n-1$-th cohomology is spanned (modulo coboundaries) by monomials $Q_{1}^{\mu_{1}} \cdots Q_{n}^{\mu_{n}}$ with $\mu_{i}<0$ and $\sum_{i=1}^{n} \mu_{i}=m$, $m \leq-n$. Thus, concretely, in the non-trivial cases, the previous isomorphism can be written in the form

$$
H^{\bullet}\left(\mathbb{P}^{n-1}, \mathcal{O}(\ell+2 m)\right) \ni Q_{1}^{\mu_{1}} \cdots Q_{n}^{\mu_{n}} \mapsto Q_{1}^{\mu_{1}} \cdots Q_{n}^{\mu_{n}} Q_{n+1}^{m} \in H^{\bullet}\left(\tilde{X}, \mathcal{O}\left(\ell D_{0}\right)\right) .
$$

2.3. Twisted rings of differential operators on $\tilde{X}$. Before discussing the twisted ring of differential operators $\mathcal{D}_{\mathcal{O}\left(\ell D_{0}\right)}(\widetilde{X})$, we consider the ring $\mathcal{D}(\bar{X})$ of global, regular differential operators on $\bar{X}$. The explicit form of this ring was computed in [8]; for a better understanding of forthcoming computations, we compute explicitly the generators of $\mathcal{D}(\bar{X})$ by using the main result of [7].

First of all, by the characterization of $\bar{X}$ as a toric variety at the beginning of Section 2, $\mathcal{D}(\bar{X})$ is spanned by differential monomials $Q^{\mu} P^{\nu}$, where (i) $\mu$ is a multiindex with $\mu_{i} \geq 0, i=1, \ldots, n$, and $\mu_{n+1} \in \mathbb{Z}$, and (ii) $\nu$ is a multiindex with non-negative components, such that $\tau:=\mu-\nu$ satisfies $\sum_{i=1}^{n} \tau_{i}-2 \tau_{n+1}=0$ (we use the same notations for the Weyl algebra as in [3]). The degree of a differential monomial $Q^{\mu} P^{\nu}$ is defined here as $|\tau|:=\sum_{i=1}^{n+1} \tau_{i}, \tau$ as above.

Lemma 2.3. The ring $\mathcal{D}(\bar{X})$ is spanned by 1 and by the differential monomials

$$
Q_{i} P_{j}, Q_{n+1} P_{n+1}, Q_{i} Q_{j} Q_{n+1}, P_{i} P_{j} Q_{n+1}^{-1}, i, j=1, \ldots, n,
$$

of degree 0, 3 and -3 respectively, subject to the relation $\sum_{i=1}^{n} Q_{i} P_{i}-2 Q_{n+1} P_{n+1}=$ 0 .

Proof. We show that any differential monomial $Q^{\mu} P^{\nu}$ as above spanning $\mathcal{D}(\bar{X})$ can be written as a linear combination of products of the above generators; for the sake of simplicity, we adopt the notation $Q^{\prime}:=\left(Q_{1}, \ldots, Q_{n}\right)$, and similarly for $P^{\prime}$ and for multi-indices $\mu^{\prime}, \nu^{\prime}$, etc. We consider a differential monomial $Q^{\mu} P^{\nu}$ of degree 0 ; then $\tau_{n+1}=0$ and $\left|\tau^{\prime}\right|=0$. Hence, we can rewrite

$$
Q^{\mu} P^{\nu}=\left(Q^{\prime}\right)^{\mu^{\prime}}\left(P^{\prime}\right)^{\nu^{\prime}} Q_{n+1}^{\mu_{n+1}} P_{n+1}^{\mu_{n+1}},
$$

and $\left(Q^{\prime}\right)^{\mu^{\prime}}\left(P^{\prime}\right)^{\nu^{\prime}}$ has degree 0 . Since $\tau_{n+1}=0$, the above conventions over the multi-indices force $\mu_{n+1} \geq 0$.

If the degree of $Q^{\mu} P^{\nu}$ is strictly positive, then $\tau_{n+1}>0$ and $\left|\tau^{\prime}\right|>0$, thus

$$
Q^{\mu} P^{\nu}=\left(Q^{\prime}\right)^{\lambda^{\prime}} Q_{n+1}^{\tau_{n+1}}\left(Q^{\prime}\right)^{\mu^{\prime \prime}}\left(P^{\prime}\right)^{\nu^{\prime \prime}} Q_{n+1}^{\nu_{n+1}} P_{n+1}^{\nu_{n+1}},
$$

where $\left|\lambda^{\prime}\right|=\left|\tau^{\prime}\right|=2 \tau_{n+1}$ and $\left|\tau^{\prime \prime}\right|=0$. We observe that, by construction, negative powers of the component $Q_{n+1}$ do not appear here.

Finally, if $Q^{\mu} P^{\nu}$ has strictly negative degree, then $\tau_{n+1}<0$. Since the component $\mu_{n+1}$ can be negative, we have to distinguish two cases, namely (i) $\mu_{n+1}<0$ and (ii) $\mu_{m+1} \geq 0$. We begin by discussing (i): Since $\tau_{n+1}<0$, then also $\left|\tau^{\prime}\right|<0$, and $Q^{\mu} P^{\nu}$ can be rewritten as

$$
Q^{\mu} P^{\nu}=\left(Q^{\prime}\right)^{\mu^{\prime \prime}}\left(P^{\prime}\right)^{\nu^{\prime \prime}} Q_{n+1}^{\nu_{n+1}} P_{n+1}^{\nu_{n+1}}\left(P^{\prime}\right)^{\lambda^{\prime}} Q_{n+1}^{\tau_{n+1}},
$$


where $\left|\lambda^{\prime}\right|=-\left|\tau^{\prime}\right|=-2 \tau_{n+1}$ and $\left|\tau^{\prime \prime}\right|=0$. As for (ii), by similar arguments, and since $\mu_{n+1} \geq 0$, the differential monomial $Q^{\mu} P^{\nu}$ can be rewritten as

$$
Q^{\mu} P^{\nu}=\left(Q^{\prime}\right)^{\mu^{\prime \prime}}\left(P^{\prime}\right)^{\nu^{\prime \prime}} Q_{n+1}^{\mu_{n+1}} P_{n+1}^{\mu_{n+1}}\left(P^{\prime}\right)^{\lambda^{\prime}} P_{n+1}^{-\tau_{n+1}},
$$

where $\left|\lambda^{\prime}\right|=-\left|\tau^{\prime}\right|=-2 \tau_{n+1}$ and $\left|\tau^{\prime \prime}\right|=0$.

Using the commutation relations of the Weyl algebra, $Q^{\mu} P^{\nu}$ can be further rewritten in all four cases as a linear combination of products of the given generators; we only observe that, in (ii), the monomial $Q^{\mu} P^{\nu}$ can be rewritten as a linear combination of products of $Q_{i} P_{i}, i=1, \ldots, n, Q_{n+1} P_{n+1}$ and $P_{i} P_{j} P_{n+1}$, $i, j=1, \ldots, n$. But the monomials $P_{i} P_{j} P_{n+1}$ equal $\left(P_{i} P_{j} Q_{n+1}^{-1}\right)\left(Q_{n+1} P_{n+1}\right)$, hence the claim follows.

Easy computations using Lemma 2.3 show that there is a surjective algebra homomorphism from $U\left(\mathfrak{s p}_{2 n}\right)$ onto $\mathcal{D}(\bar{X})$ (see also [6] and [7); this surjection is explicitly given in terms of Chevalley-Cartan generators of $\mathfrak{s p}_{2 n}$ by the formulæ

$$
\begin{aligned}
e_{i} & = \begin{cases}-Q_{i+1} P_{i}, & i=1, \ldots, n-1, \\
\frac{1}{2} P_{n}^{2} Q_{n+1}^{-1}, & i=n,\end{cases} \\
h_{i} & = \begin{cases}-Q_{i} P_{i}+Q_{i+1} P_{i+1}, & i=1, \ldots, n-1, \\
-Q_{n} P_{n}-\frac{1}{2}, & i=n,\end{cases} \\
f_{i} & = \begin{cases}-Q_{i} P_{i+1}, & i=1, \ldots, n-1, \\
-\frac{1}{2} Q_{n}^{2} Q_{n+1} .\end{cases}
\end{aligned}
$$

We consider, for an integer $\ell$, the twisted ring of differential operators $\mathcal{D}_{\mathcal{O}\left(\ell D_{0}\right)}^{r}(\widetilde{X})$ with rational coefficients; there is a well-defined homomorphism

$$
U(\mathfrak{g}) \stackrel{\psi_{\ell}}{\rightarrow} \mathcal{D}_{\mathcal{O}\left(\ell D_{0}\right)}^{r}(\widetilde{X}),
$$

which is induced by the formulæ (2).

Remark 2.4. The ring $\mathcal{D}_{\mathcal{O}\left(\ell D_{0}\right)}^{r}(\widetilde{X})$ is well defined, as it is viewed as the ring of global sections of the twisted sheaf of differential operators on $\mathcal{O}\left(\ell D_{0}\right)$ with rational coefficients; the latter sheaf is obtained by tensoring the twisted sheaf of differential operators $\mathcal{O}\left(\ell D_{0}\right)$ by the sheaf of rational functions on $\widetilde{X}$ over the structure sheaf of $\widetilde{X}$. The relation $\sum_{i=1}^{n} Q_{i} P_{i}-2 Q_{n+1} P_{n+1}-\ell=0$ in $\mathcal{D}_{\mathcal{O}\left(\ell D_{0}\right)}(\widetilde{X})$ (see Lemma2.5) obviously holds true in $\mathcal{D}_{\mathcal{O}\left(\ell D_{0}\right)}^{r}(\widetilde{X})$ also.

Similarly to Lemma 2.3, the toric structure of $\widetilde{X}$ permits us to compute explicit generators for $\mathcal{D}_{\mathcal{O}\left(\ell D_{0}\right)}(\widetilde{X})$.

Lemma 2.5. For any integer $\ell$, the ring $\mathcal{D}_{\mathcal{O}\left(\ell D_{0}\right)}(\tilde{X})$ is spanned by 1 and by the differential monomials

$$
Q_{i} P_{j}, Q_{n+1} P_{n+1}, Q_{i} Q_{j} Q_{n+1}, P_{i} P_{j} P_{n+1}, i, j=1, \ldots, n,
$$

of degree 0,3 and -3 respectively, subject to the relation $\sum_{i=1}^{n} Q_{i} P_{i}-2 Q_{n+1} P_{n+1}-$ $\ell=0$.

Proof. $\mathcal{D}_{\mathcal{O}\left(\ell D_{0}\right)}(\widetilde{X})$ is spanned by differential monomials $Q^{\mu} P^{\nu}$, where $\mu, \nu$ are now both multi-indices with non-negative components such that $\tau=\mu-\nu$ satisfies $\sum_{i=1}^{n} \tau_{i}-2 \tau_{n+1}=0$. The proof now follows essentially along the same lines of 
the proof of Lemma 2.3, the only difference being that, when considering differential monomials of negative degree, we only have to consider the case (ii), since $\mu_{n+1} \geq 0$.

We recall the parabolic triangular decomposition of $\mathfrak{g}$,

$$
\mathfrak{g}=\mathfrak{p} \oplus \mathfrak{r}_{-}=\mathfrak{r}_{+} \oplus \mathfrak{m} \oplus \mathfrak{r}_{-},
$$

where $\mathfrak{m} \cong \mathfrak{g l}_{n}$ is the reductive part of $\mathfrak{p}$, with semisimple part $\mathfrak{s l}_{n}$ and 1-dimensional center $\mathfrak{z} ; \mathfrak{r}_{+}$is the abelian nilradical, and $\mathfrak{r}_{-}$is abelian and an $\mathfrak{m}$-module. In terms of differential operators, direct computations imply that the homomorphic images of $\mathfrak{m}$, resp. $\mathfrak{r}_{-}$, w.r.t. (3) are spanned by the monomials $Q_{i} P_{j}, i, j=$ $1, \ldots, n$ and $Q_{n+1} P_{n+1}$, resp. $Q_{i} Q_{j} Q_{n+1}, i, j=1, \ldots, n$, with the additional relation $\sum_{i=1}^{n} Q_{i} P_{i}-2 Q_{n+1} P_{n+1}-\ell=0$; obviously, these two subspaces lie in $\mathcal{D}_{\mathcal{O}\left(\ell D_{0}\right)}(\widetilde{X})$. On the other hand, e.g. by Subsection 3.2, Remark (vii) of [6] there is a canonical generator $z$ of $\mathfrak{z}$, which satisfies the commutation relations

$$
[z, x]=x, \quad x \in \mathfrak{r}_{+}, \quad[z, y]=-y, \quad y \in \mathfrak{r}_{-} .
$$

The image of the generator $z$ w.r.t. (3) is

$$
\psi_{\ell}(z)=-\frac{1}{2} \sum_{i=1}^{n} Q_{i} P_{i}-\frac{n}{4}=-Q_{n+1} P_{n+1}-\frac{\ell}{2}-\frac{n}{4}
$$

in $\mathcal{D}_{\mathcal{O}\left(\ell D_{0}\right)}(\widetilde{X})$. We denote from now on by $z_{\ell}$ the central element $z+\frac{\ell}{2}+\frac{n}{4}$ of $U(\mathfrak{g})$. We finally consider the differential monomials $P_{i} P_{j} P_{n+1}$ in $\mathcal{D}_{\mathcal{O}\left(\ell D_{0}\right)}^{r}(\widetilde{X})$; the relation

$$
P_{i} P_{j} P_{n+1}=\left(-P_{i} P_{j} Q_{n+1}^{-1}\right)\left(-Q_{n+1} P_{n+1}\right)
$$

holds true. Direct computations using Lemma 2.3 show that the differential monomials $P_{i} P_{j} Q_{n+1}^{-1}$ lie in the homomorphic image of $\mathfrak{r}_{+}$w.r.t. (3), while $Q_{n+1} P_{n+1}$ lies in the homomorphic image of $\mathfrak{z} \oplus \mathbb{C}$; it follows that the homomorphic image w.r.t. (3) of the subspace $\mathfrak{r}_{+}^{\ell}$ of $U(\mathfrak{g})$ spanned by elements of the form $x z_{\ell}$, where $x$ is in $\mathfrak{r}_{+}$, lies in $\mathcal{D}_{\mathcal{O}\left(\ell D_{0}\right)}(\tilde{X})$ by Lemma 2.5 and corresponds to the vector subspace spanned by the differential monomials $P_{i} P_{j} P_{n+1}$.

Thus, combining these arguments with Lemma 2.5, the homomorphism (3), restricted to the associative subalgebra $\mathfrak{A}_{\ell}$ of $U(\mathfrak{g})$ generated by $1, \mathfrak{m}, \mathfrak{r}_{-}$and $\mathfrak{r}_{+}^{\ell}$ descends to a surjective homomorphism onto $\mathcal{D}_{\mathcal{O}\left(\ell D_{0}\right)}(\widetilde{X})$.

Theorem 2.6. For any integer $\ell$, there exists an associative subalgebra $\mathfrak{A}_{\ell}$ of $U(\mathfrak{g})$ and a surjective homomorphism from $\mathfrak{A}_{\ell}$ to the twisted algebra $\mathcal{D}_{\mathcal{O}\left(\ell D_{0}\right)}(\widetilde{X})$.

\section{Representation theory of the Algebra $\mathfrak{A}_{\ell}$}

For a given integer $\ell$, the algebra $\mathfrak{A}_{\ell}$, defined at the end of Subsection 2.3, contains a copy of the semisimple part of $\mathfrak{m}$, which is simply $\mathfrak{s l}_{n}$. We have thus the additional relation in $\mathfrak{A}_{\ell}$

$$
\left[z_{\ell}, x z_{\ell}\right]=x z_{\ell}
$$

for any $x \in \mathfrak{r}_{+}$. We observe that the nilradical $\mathfrak{r}_{+}$is isomorphic to the finitedimensional, irreducible highest weight $\mathfrak{s l}_{n}$-module $L\left(2 \varpi_{1}\right)$, with highest weight vector $e_{\beta}$ and highest weight $\beta, \beta$ being the highest root of $\mathfrak{g}$; moreover, $\mathfrak{r}_{+} \cong \mathfrak{r}_{-}$ as a vector space, while, as an $\mathfrak{m}$-module, it is isomorphic to the finite-dimensional, irreducible highest weight module $L\left(2 \varpi_{n-1}\right)$. On the other hand, using again the 
Cartan involution on $\mathfrak{s l}_{n}, \mathfrak{r}_{+}$can be viewed as the finite-dimensional, irreducible lowest weight module $L\left(-2 \varpi_{n-1}\right)$, with lowest weight vector $e_{\alpha_{n}}$ and lowest weight $\alpha_{n}$; similar results hold true for $\mathfrak{r}_{-}$.

3.1. A natural "universal" parabolic subalgebra of $\mathfrak{A}_{\ell}$. We consider the vector subspace $\mathfrak{p}_{\ell}$ of $\mathfrak{A}_{\ell} \subset U(\mathfrak{g})$ spanned by $\mathfrak{m}$ and $\mathfrak{r}_{+}^{\ell}$.

Lemma 3.1. The vector space $\mathfrak{p}_{\ell}$ inherits from $U(\mathfrak{g})$ the structure of a Lie algebra; furthermore, as a Lie algebra, $\mathfrak{p}_{\ell}$ is isomorphic to the parabolic subalgebra $\mathfrak{p}$ of $\mathfrak{g}$.

Proof. The Lie algebra structure is inherited from the associative structure of $U(\mathfrak{g})$ : since $\mathfrak{r}_{+}$is an $\mathfrak{m}$-module and $z_{\ell}$ commutes in $U(\mathfrak{g})$ with $\mathfrak{m}$, the only non-trivial statement to prove is that $\mathfrak{r}_{+} z_{\ell}$ is abelian. We consider $x, x^{\prime}$ in $\mathfrak{r}_{+}$; hence

$$
\begin{aligned}
{\left[x z_{\ell}, x^{\prime} z_{\ell}\right] } & =\left[x z_{\ell}, x^{\prime}\right] z_{\ell}+x^{\prime}\left[x z_{\ell}, z_{\ell}\right] \\
& =x\left[z_{\ell}, x^{\prime}\right] z_{\ell}+\left[x, x^{\prime}\right] z_{\ell}^{2}+x^{\prime} x\left[z_{\ell}, z_{\ell}\right]+x^{\prime}\left[x, z_{\ell}\right] z_{\ell} \\
& =x x^{\prime} z_{\ell}-x^{\prime} x z_{\ell}=\left[x, x^{\prime}\right] z_{\ell}=0,
\end{aligned}
$$

where we used that $\mathfrak{r}_{+}$is an abelian Lie algebra. The Lie algebra isomorphism $\mathfrak{p} \cong \mathfrak{p}_{\ell}$ is simply given by

$$
\mathfrak{m} \ni m \mapsto m \in \mathfrak{p}_{\ell}, \quad r_{+} \ni x \mapsto x z_{\ell}
$$

As a result, for any integer $\ell$, the algebra $\mathfrak{A}_{\ell}$ contains a copy of the parabolic subalgebra $\mathfrak{p} \subset \mathfrak{g}$; filtration arguments à la Poincaré-Birkhoff-Witt show that this induces an isomorphism between $U(\mathfrak{p})$ and the subalgebra of $\mathfrak{A}_{\ell}$ spanned by $\mathfrak{m}$ and $\mathfrak{r}_{+}^{\ell}$. We observe that, in spite of Theorem 2.6 of Subsection 2.3. $\mathfrak{p} \cong \mathfrak{p}_{\ell}$ is the vector space spanned by $Q_{i} P_{j}, Q_{n+1} P_{n+1}$ and $P_{i} P_{j} P_{n+1}, i, j=1, \ldots, n$.

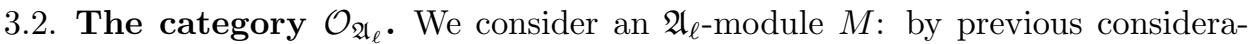
tions, $M$ inherits the structure of a $U\left(\mathfrak{s l}_{n}\right)$-module. An element of the dual of $\mathfrak{z} \ell$, the 1-dimensional subspace spanned by $z_{\ell}$, is said to be a weight for $\mathfrak{A}_{\ell}$; it is simply

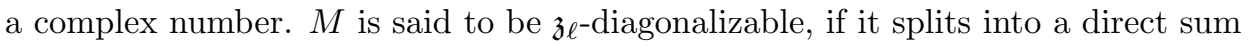

$$
M=\bigoplus_{\lambda \in \mathfrak{z}_{\ell}^{*}} M^{\lambda}, \quad M^{\lambda}=\left\{v \in M: z_{\ell} v=\lambda v\right\}
$$

A weight of $M$ is a weight $\lambda$ with non-trivial weight subspace $M^{\lambda}$. We introduce a partial order on $\mathfrak{z}_{\ell}^{*}$ via

$$
\mu \leq \nu \Leftrightarrow \nu-\mu \in \mathbb{N}
$$

Previous computations yield

$$
\left(x z_{\ell}\right)\left(M^{\lambda}\right) \subseteq M^{\lambda+1}, \quad y\left(M^{\lambda}\right) \subseteq M^{\lambda-1}, \quad m\left(M^{\lambda}\right) \subseteq M^{\lambda},
$$

for $x \in \mathfrak{r}_{+}, y \in \mathfrak{r}_{-}$and $m \in \mathfrak{m}$.

We define the category $\mathcal{O}_{\mathfrak{A}_{\ell}}$ by the following requirements: an object of $\mathcal{O}_{\mathfrak{A}_{\ell}}$ is an $\mathfrak{A}_{\ell}$-module such that

i) $M$ is a $\mathfrak{z} \ell^{- \text {diagonalizable }} \mathfrak{A}_{\ell \text {-module; }}$

ii) every weight subspace $M^{\lambda}$ of $M$ is finite-dimensional;

iii) there exists a weight $\mu$, such that all weights $\lambda$ of $M$ satisfy $\lambda \leq \mu$; hence, $\mu$ is called a highest weight. 
By standard arguments about gradations, the category $\mathcal{O}_{\mathfrak{A}_{\ell}}$ is closed w.r.t. taking submodules and quotient modules.

Observe that for the weight subspace to the highest weight $\mu$ of an object $M$ of $\mathcal{O}_{\mathfrak{A}_{\ell}}$, we have $\left(\mathfrak{r}_{+}^{\ell}\right) M^{\mu}=0$. Furthermore, every weight subspace $M^{\lambda}$ of $M$ in $\mathcal{O}_{\mathfrak{A}_{\ell}}$ has the structure of an $\mathfrak{s l}_{n}$-module.

Since every weight subspace $M^{\lambda}$ of $M$ is finite-dimensional, it splits into a finite direct sum of irreducible, finite-dimensional $\mathfrak{s l}_{n}$-modules $L(\nu):=L_{\mathfrak{s l}_{n}}(\nu)$, for $\nu$ a dominant weight, possibly depending on the $\mathfrak{z}$-weight $\lambda$. In particular, every weight subspace $M^{\lambda}$ of an object $M$ of $\mathcal{O}_{\mathfrak{A}_{\ell}}$ contains finitely many highest weight vectors w.r.t. the $\mathfrak{s l}_{n}$-action; if, moreover, $\lambda$ is a highest weight w.r.t. $\mathfrak{z} \ell$, such vectors are also annihilated by $\mathfrak{r}_{+}^{\ell}$. As a consequence, an object $M$ of $\mathcal{O}_{\mathfrak{A}_{\ell}}$ possesses finitely many highest weight vectors $v$ for $\mathfrak{A}_{\ell}$, i.e. vectors $v$ in $M$ satisfying

$$
e_{\alpha_{i}} v=0, i=1, \ldots, n-1,\left(e_{\alpha_{n}} z_{\ell}\right) v=0, z_{\ell} v=\mu z, h v=\nu(h) v,
$$

where $h$ belongs to the Cartan subalgebra $\mathfrak{h}$ of $\mathfrak{s l}_{n}$, and $\nu$ is a weight for $\mathfrak{h}$.

Lemma 3.2. If $v$ is a highest weight vector of $M$ in $\mathcal{O}_{\mathfrak{A}_{\ell}}$, the vectors $e_{\alpha_{n}}^{m} v$, for any positive integer $m$, constitute a sequence of $\mathfrak{s l}_{n}$-primitive weight vectors in $M^{\mu-m}$, of dominant weight $\nu-\left.m \alpha_{n}\right|_{\mathfrak{h}}$.

Proof. The proof follows by a standard induction argument; the main point is that any bracket $\left[e_{\alpha_{i}}, e_{-\alpha_{n}}\right], i=1, \ldots, n-1$, vanishes, since $-\alpha_{n}+\alpha_{i}$ does not belong to the root system of $\mathfrak{g}$. Notice that $\left.\alpha_{n}\right|_{\mathfrak{h}}=-2 \varpi_{n-1}$, hence, if $\nu$ is a dominant weight for $\mathfrak{s l}_{n}$, then so is $\nu-\left.m \alpha_{n}\right|_{\mathfrak{h}}$, for any positive integer $m$. We only finally observe that the vectors $e_{-\alpha_{n}}^{m} v$ can also be trivial.

Special objects of the category $\mathcal{O}_{\mathfrak{A}_{\ell}}$ are modules, for which every weight subspace $M^{\lambda}$ is irreducible as an $\mathfrak{s l}_{n}$-module; as a consequence, to every weight subspace $M^{\lambda}$ belongs a unique dominant weight $\nu$ (which possibly depends on $\lambda$ ), such that $M^{\lambda}=L(\nu)$. If e.g. $v$ is a highest weight vector of such an object $M$ of $\mathcal{O}_{\mathfrak{A}_{\ell}}$, and if $e_{-\alpha_{n}}^{m} v \neq 0$, for any positive integer $m$, then we have

$$
M=\bigoplus_{\lambda \leq \mu} M^{\lambda}, \quad M^{\lambda} \cong L\left(\nu-\left.(\mu-\lambda) \alpha_{n}\right|_{\mathfrak{h}}\right),
$$

which will happen for most of the examples discussed in Subsection 5.2. Since the category $\mathcal{O}_{\mathfrak{A}_{\ell}}$ is closed w.r.t. taking submodules, a non-trivial submodule $N$ of $M$ in $\mathcal{O}_{\mathfrak{A}_{\ell}}$ possesses a primitive subspace, i.e. there is a weight $\lambda$ of $M$, such that $N^{\lambda}=N \cap M^{\lambda}$ is annihilated by $\mathfrak{r}_{+}^{\ell}$; obviously, $N^{\lambda}$ is an $\mathfrak{s l}_{n}$-submodule of $M^{\lambda}$. On the other hand, if for an object $M$ of $\mathcal{O}_{\mathfrak{A}_{\ell}}$, there exists a weight $\lambda$ and an $\mathfrak{s l}_{n}$-submodule $N^{\lambda} \subseteq M^{\lambda}$, which is annihilated by $\mathfrak{r}_{+}^{\ell}$, then $M$ is reducible; we consider the $\mathfrak{A}_{\ell}$-module $N$ generated by $N^{\lambda}$, which is obviously a submodule of $M$ and belongs to the category $\mathcal{O}_{\mathfrak{A}_{\ell}}$. Therefore, the irreducibility of objects of $\mathcal{O}_{\mathfrak{A}_{\ell}}$ is in one-to-one correspondence with the existence of primitive weight subspaces, i.e. $\mathfrak{s l}_{n}$-submodules of some weight subspaces, which are annihilated by the action of $\mathfrak{r}_{+}^{\ell}$.

Next, the category $\mathcal{O}_{\mathfrak{A}_{\ell}}$ is "too big"; in fact, as the next theorem shows, many objects of $\mathcal{O}_{\mathfrak{A}_{\ell}}$ can be regarded as $\mathfrak{g}$-modules.

Theorem 3.3. If the highest weight $\mu$ in the weight decomposition of an object $M$ of the category $\mathcal{O}_{\mathfrak{A}_{\ell}}$ does not belong to $\mathbb{N}$, then $M$ lifts to a $\mathfrak{g}$-module. 
Proof. Every weight $\lambda$ in the weight decomposition of $M$ is non-zero, if $\mu$ does not belong to $\mathbb{N}$, since the weights of $M$ are all less than or equal to $\mu$ w.r.t. the above partial order. We define a $\mathfrak{g}$-action on $M$ by defining it on any weight subspace $M^{\lambda}$ of $M$ :

$$
x v:=\frac{1}{\lambda}\left(x z_{\ell}\right) v, m v:=m v, y v:=y v, x \in \mathfrak{r}_{+}, m \in \mathfrak{m}, y \in \mathfrak{r}_{-}, v \in M^{\lambda} .
$$

We have to show that the previous formulæ define a true action; the only non-trivial relations to prove are

$$
[m, x] v=m(x v)-x(m v) \text { and }[x, y] v=x(y v)-y(x v),
$$

for any $x \in \mathfrak{r}_{+}, m \in \mathfrak{m}, y \in \mathfrak{r}_{-}$and $v \in M^{\lambda}$, and any weight $\lambda$ in the weight decomposition. Since $\mathfrak{r}_{+}^{\ell}$ is an $\mathfrak{m}$-module by Lemma 3.1, then

$$
\begin{aligned}
{[m, x] v } & =\frac{1}{\lambda}\left([m, x] z_{\ell}\right) v=\frac{1}{\lambda}\left[m, x z_{\ell}\right] v \\
& =\frac{1}{\lambda} m\left(\left(x z_{\ell}\right) v\right)-\frac{1}{\lambda}\left(x z_{\ell}\right)(m v) \\
& =m(x v)-x(m v),
\end{aligned}
$$

since the action of $\mathfrak{m}$ preserves the weight $\lambda$, and by the obvious relation $\left[m, x z_{\ell}\right]=$ $[m, x] z_{\ell}$ in $\mathfrak{A}_{\ell}$. As for the second relation, we have

$$
\begin{aligned}
x(y v)-y(x v) & =\frac{1}{\lambda-1}\left(x z_{\ell}\right)(y v)-\frac{1}{\lambda} y\left(\left(x z_{\ell}\right) v\right) \\
& =\frac{1}{\lambda(\lambda-1)}\left(\lambda\left(x z_{\ell}\right)(y v)-(\lambda-1) y\left(\left(x z_{\ell}\right) v\right)\right) \\
& =\frac{1}{\lambda(\lambda-1)}\left(\lambda\left(x z_{\ell}\right)(y v)-(\lambda-1)\left[y, x z_{\ell}\right] v-(\lambda-1)\left(x z_{\ell}\right)(y v)\right) \\
& =\frac{1}{\lambda(\lambda-1)}\left(-(\lambda-1)\left[y, x z_{\ell}\right] v+\left(x z_{\ell}\right)(y v)\right) .
\end{aligned}
$$

Since $\mathfrak{A}_{\ell}$ is a subalgebra of $U(\mathfrak{g})$, we have the relations in $\mathfrak{A}_{\ell}$ :

$$
\left[y, x z_{\ell}\right]=-[x, y] z_{\ell}+x y,\left(z_{\ell}-1\right)(x y)=\left(x z_{\ell}\right) y ;
$$

the latter can also be rewritten in the form

$$
(x y) v=\frac{1}{\lambda-1}\left(x z_{\ell}\right)(y v), \quad v \in M^{\lambda}, \quad \lambda, \lambda-1 \neq 0 .
$$

Finally, using these relations, we have

$$
\begin{aligned}
& \frac{1}{\lambda(\lambda-1)}\left(-(\lambda-1)\left[y, x z_{\ell}\right] v+\left(x z_{\ell}\right)(y v)\right) \\
& \quad=\frac{1}{\lambda}[x, y]\left(z_{\ell} v\right)-\frac{1}{\lambda}(x y) v+\frac{1}{\lambda(\lambda-1)}\left(x z_{\ell}\right)(y v)=[x, y] v .
\end{aligned}
$$

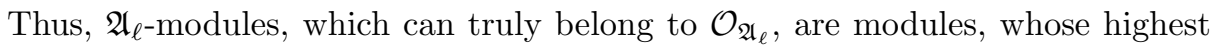
weight $\mu$ w.r.t. $\mathfrak{z} \ell$ is a positive integer. If $M$ is such a module, the sequence of weights of $M$ is contained in an arithmetic sequence of the form $\mu-n, n \in \mathbb{N}$, with $\mu$ a positive integer; as we will see in forthcoming examples, the sequence of weights can be infinite or can contain only finitely many terms.

Assume finally that every weight subspace $M^{\lambda}$ of such a module $M$ is an irreducible $\mathfrak{s l}_{n}$-module and that the highest weight $\mu$ of $M$ is non-negative. Then the maximal non-trivial submodule of $M$ is associated to the maximal weight $\lambda$ of $M$, 
such that $M^{\lambda}$ is annihilated by $\mathfrak{r}_{+}^{\ell}$. Namely, any submodule $N$ of $M$ is associated to a non-trivial $\mathfrak{s l}_{n}$-submodule of $M^{\lambda}$, for some weight $\lambda$ of $M$, which coincides with $M^{\lambda}$, since $M^{\lambda}$ is irreducible; thus, the corresponding submodule $N$ is $\bigoplus_{\lambda^{\prime}<\lambda} M^{\lambda^{\prime}}$. This fact implies that, choosing $\lambda$ to be maximal among all weights of $\bar{M}$ such that $M^{\lambda}$ is annihilated by $\mathfrak{r}_{+}^{\ell}$, the submodule $N:=\bigoplus_{\lambda^{\prime}<\lambda} M^{\lambda^{\prime}}$ is the maximal non-trivial submodule of $M$. Therefore, the quotient module $M / N$ is the unique irreducible finite-dimensional quotient module of $M$ of highest weight $\mu \in \mathbb{N}$ w.r.t. $\mathfrak{z} \ell ;$ to the highest weight $\mu$ there corresponds a unique dominant highest weight $\nu$ for $M^{\mu}$ as a $\mathfrak{s l}_{n}$-module, such that $M^{\mu}=L(\nu)$.

\section{Weighted PROJECTIVE SPACES}

4.1. Weighted projective spaces via $I$-reflections. We consider the regular fan $\widetilde{\Delta}$ of the resolution of singularities $\widetilde{X}$ and the $I$-reflection (see [3] and [9])

$$
k_{i} \mapsto \begin{cases}k_{i}, & i=1, \ldots, n, \\ -k_{i}, & i=n+1 .\end{cases}
$$

This $I$-reflection determines generating vectors $k_{i}^{\prime}$ and a corresponding fan $\Delta^{\prime}$, whose cones of maximal dimension are spanned by exactly $n$ out of the $k_{i}^{\prime}$. Observe that it is no longer a regular fan, since the cone associated to $k_{1}^{\prime}, \ldots, k_{n}^{\prime}$ is not spanned by (a part of) a basis of $N$; the generating vectors $k_{i}^{\prime}$ satisfy the relation $\sum_{i=1}^{n} k_{i}^{\prime}+2 k_{n+1}^{\prime}=0$. Following [8], the toric variety $Y$ associated to $\Delta^{\prime}$ is the algebro-geometric quotient $\mathbb{A}^{n+1} \backslash\{0\} / \mathbb{C}^{\times}$, with torus action

$$
\mathbb{C}^{\times} \times \mathbb{A}^{n+1} \backslash\{0\} \ni\left(u, Q_{1}, \ldots, Q_{n+1}\right) \mapsto\left(u Q_{1}, \ldots, u Q_{n}, u^{2} Q_{n+1}\right) \in \mathbb{A}^{n+1} \backslash\{0\} .
$$

Therefore, the $n$-dimensional weighted projective space $Y=\mathbb{P}^{n}(1, \ldots, 1,2)$ is related to the resolution of singularities $\widetilde{X}$ of the irreducible component $\bar{X}$ of $\bar{O}_{\min } \cap \mathfrak{n}_{+}$ by an $I$-reflection.

4.2. Cohomology of weighted projective spaces. The weighted projective space $Y$ can be viewed as the projective spectrum of the weighted polynomial ring $S=\mathbb{C}\left[Q_{1}, \ldots, Q_{n+1}\right]$, with degrees $\operatorname{deg} Q_{i}=1$, if $i=1, \ldots, n$, and $\operatorname{deg} Q_{n+1}=2$; thus, the component $S_{d}$ of degree $d$ is spanned by monomials $Q^{\mu}$, with the multiindex $\mu$ satisfying $\sum_{i=1}^{n} \mu_{i}+2 \mu_{n+1}=d$. By construction, $Y$ is covered by $n+1$ open affine subsets, corresponding to $\left\{Q_{i} \neq 0\right\}$; the affine subsets corresponding to $\left\{Q_{i} \neq 0\right\}, i=1, \ldots, n$, are isomorphic to the affine space $\mathbb{A}^{n}$, and the subset $\left\{Q_{n+1} \neq 0\right\}$ is isomorphic to $\bar{X}$ as in Subsection 2.1.

In complete analogy with the theory of usual projective spaces, the Serre sheaf $\mathcal{O}(\ell)$ on $Y$, for any integer $\ell$, is the sheaf associated to the shifted graded ring $S[\ell]$ ([5, Proposition 5.11 and Definition right before). The main difference with the usual projective space is that $\mathcal{O}(\ell)$ is invertible exactly when $\ell$ is even, since $S$ is generated over $S_{0}=\mathbb{C}$ by $S_{1}$ and $S_{2}$. Using the toric structure of $Y$, we can compute the Picard group of $Y$ and $A_{n-1}(Y)$ : the group $A_{n-1}(Y)$ of Weil divisors on $X$ modulo linear equivalence is isomorphic to $\mathbb{Z}$ and is generated, say, by the divisor $D_{1}$. More precisely, $A_{n-1}(Y)$ is spanned over $\mathbb{Z}$ by the Weil divisors $D_{i}$, subject to the relations $D_{i} \sim D_{j}, i, j=1, \ldots, n$, and $2 D_{i} \sim D_{n+1}, i=1, \ldots, n$. On the other hand, by direct computations involving Cartier divisors, the Picard group of $Y$ is generated over $\mathbb{Z}$ by the Weil divisor $2 D_{1}$. Observe the obvious 
identification $\mathcal{O}\left(\ell D_{0}\right)=\mathcal{O}(\ell)$, for any even integer $\ell$; hence, the Serre bundle $\mathcal{O}(2)$ is the generator of the Picard group of $Y$.

Theorem 4.1. The cohomology of $Y$ with values in any sheaf $\mathcal{O}(\ell)$, for any integer $\ell$, is concentrated in degree 0 and $n$; more explicitly, we have

$$
\bigoplus_{\ell \in \mathbb{Z}} H^{0}(Y, \mathcal{O}(\ell)) \cong S, \quad H^{n}(Y, \mathcal{O}(-n-2)) \cong \mathbb{C},
$$

and there is a perfect pairing

$$
H^{n}(Y, \mathcal{O}(\ell)) \otimes H^{0}(Y, \mathcal{O}(-\ell-n-2)) \rightarrow H^{n}(Y, \mathcal{O}(-n-2)) \cong \mathbb{C} .
$$

Proof. The proof goes along the same lines as the proof of Theorem 5.1, pages 225228 , of [5. The only difference is that we have to keep track of different degrees in the polynomial ring defining $Y$, which produces different shifts for $\ell$ in the above pairing.

As a consequence, $H^{0}(X, \mathcal{O}(\ell))$ is non-trivial exactly for $\ell \geq 0$; on the other hand, $H^{n}(X, \mathcal{O}(\ell))$ is spanned by negative monomials $Q_{1}^{\mu_{1}} \cdots Q_{n+1}^{\mu_{n+1}}$, where $\mu_{i}<0$ and $\sum_{i=1}^{n} \mu_{i}+2 \mu_{n+1}=\ell$ (modulo coboundaries), for $\ell \leq-n-2$. The generator of $H^{n}(X, \mathcal{O}(-n-2))$ is thus $Q_{1}^{-1} \cdots Q_{n+1}^{-1}$, and the perfect pairing is given by the same formula as in the proof of Theorem 5.1 of 5 .

\section{Fourier transforms AND highest Weight MOdUles}

5.1. Fourier transforms, weighted projective spaces and resolution of singularities. We refer to Section 3 of [3] for notation and for the Main Theorem. From Section 4 , the fan of the weighted projective space $Y$ is not regular; hence, Theorem 3.2 of $[3$ requires more care, as observed in Remark 3.3 of [3]. Namely, we have to determine the Cartier divisors on $\widetilde{X}$, whose image w.r.t. $\phi_{I}$ ( $\phi_{I}$ is defined in [3. Lemma 3.1) are Cartier divisors in $Y$. As seen before, the Picard group of $\widetilde{X}$ is generated over $\mathbb{Z}$ by the divisor $D_{0}$, and the image w.r.t. $\phi_{I}$ of a general Cartier divisor $\ell D_{0}$, for an integer $\ell$, is readily computed

$$
\phi_{I}\left(\ell D_{0}\right)=\ell D_{1}^{\prime}-D_{n+1}^{\prime} \sim(\ell-2) D_{1}^{\prime},
$$

where $D_{i}$ and $D_{i}^{\prime}$ are $T$-Weil divisors of $\widetilde{X}$ and $Y$ respectively; by results of Subsection $4.2, \ell$ must be even. Thus, Theorem 3.2 of [3] yields the following.

Theorem 5.1. Using the notation of Section 3 of [3], for any even integer $\ell$, the Fourier transform $F_{I}$ determines an isomorphism

$$
\mathcal{D}_{\mathcal{O}\left(\ell D_{0}\right)}(\widetilde{X}) \rightarrow \mathcal{D}_{\mathcal{O}(\ell-2)}(Y) .
$$

Remark 5.2. A partial version of the isomorphism of Theorem 5.1 (without geometric insights) appears in [10, Corollary 2.3, where the author discusses similar rings of differential operators.

Combining Theorem 5.1 with the results of Subsection 2.2 gives

Corollary 5.3. For any even integer $\ell$, there is a surjective algebra homomorphism

$$
\mathfrak{A}_{\ell+2} \rightarrow \mathcal{D}_{\mathcal{O}(\ell)}(Y) .
$$


5.2. The module structure on cohomology. By Theorem 2.6 from Subsection 2.2. the cohomology groups of $\widetilde{X}$ with values in $\mathcal{O}\left(\ell D_{0}\right)$, for any integer $\ell$, are $\mathfrak{A}_{\ell}$-modules. Hence, Corollary 5.3 endows the cohomology groups of $Y$ with values in $\mathcal{O}(\ell)$, for an even integer $\ell$, with the structure of $\mathfrak{A}_{\ell+2}$-modules. We now proceed to analyze the respective module structures with the tools of Section 3

Remark 5.4. In Theorems 5.5, 5.6 and 5.8 of Subsubsection5.2.1, and Theorems 5.9 and 5.10 of Subsubsection 5.2.2, the notation $L(\bullet)$ for the unique irreducible subquotient of a certain Verma module appears frequently, and in certain cases it refers to $\mathfrak{s l}_{n}$, while in some other cases to $\mathfrak{s p}_{2 n}$; we will use a subscript with the corresponding Lie algebra in order to avoid confusion.

5.2.1. The module structure on the cohomology of $\widetilde{X}$. The 0-th cohomology group $H^{0}\left(\widetilde{X}, \mathcal{O}\left(\ell D_{0}\right)\right)$ is always non-trivial and infinite-dimensional; it is spanned by monomials $Q^{\mu}$, for multi-indices $\mu$ satisfying $\sum_{i=1}^{n} \mu_{i}-2 \mu_{n+1}=\ell$. The $n-1$ th cohomology group $H^{n-1}\left(\widetilde{X}, \mathcal{O}\left(\ell D_{0}\right)\right)$ is non-trivial exactly for $\ell \leq-n$; it is spanned (modulo coboundaries) by monomials $Q^{\mu}$, where $\mu_{i}<0, i=1, \ldots, n$, $\mu_{n+1} \geq 0$, and $\sum_{i=1}^{n} \mu_{i}-2 \mu_{n+1}=\ell$.

Theorem 5.5. For any positive integer $\ell$, there is a weight space decomposition

$$
H^{0}\left(\widetilde{X}, \mathcal{O}\left(\ell D_{0}\right)\right) \cong \bigoplus_{\lambda \leq 0} L_{\mathfrak{s l}_{n}}\left((\ell-2 \lambda) \varpi_{n-1}\right)
$$

in the category $\mathcal{O}_{\mathfrak{A}_{\ell}}$; the module $H^{0}\left(\widetilde{X}, \mathcal{O}\left(\ell D_{0}\right)\right)$ is irreducible and is generated by the highest weight vector of the subspace of weight 0 w.r.t. $\mathfrak{z} \ell$.

Proof. By results of Subsection 3.2 , we need to show that $M=H^{0}\left(\widetilde{X}, \mathcal{O}\left(\ell D_{0}\right)\right)$ is in the category $\mathcal{O}_{\mathfrak{A}_{\ell}}$. The generator of $\mathfrak{z} \ell$ corresponds to $-Q_{n+1} P_{n+1}$; it is obvious that the decomposition from Theorem 2.2 corresponds to the weight decomposition

$$
H^{0}\left(\widetilde{X}, \mathcal{O}\left(\ell D_{0}\right)\right)=\bigoplus_{\lambda \leq 0} M^{\lambda}, \quad M^{\lambda}=H^{0}\left(\mathbb{P}^{n-1}, \mathcal{O}(\ell-2 \lambda)\right) .
$$

The weight subspaces $M^{\lambda}$ are finite-dimensional; the sequence of weights coincides with the non-positive integers. Observe that the highest weight w.r.t. $\mathfrak{z} \ell$ belongs to $\mathbb{N}$. Every weight subspace $M^{\lambda}$ is a finite-dimensional irreducible $\mathfrak{s l}_{n}$-module, with highest weight vector $Q_{n}^{\ell-2 \lambda} Q_{n+1}^{-\lambda}$ and corresponding highest weight $(\ell-2 \lambda) \varpi_{n-1}$, whence $M^{\lambda}=L_{\mathfrak{s l}_{n}}\left((\ell-2 \lambda) \varpi_{n-1}\right)$. To show irreducibility, it remains to prove that the only primitive subspace of $M$ is $M^{0}$. To this purpose, we need to compute the action of $\mathfrak{r}_{+}^{\ell}$, which is spanned by the differential operators $P_{i} P_{j} P_{n+1}, i, j=$ $1, \ldots, n$ on $M$. It suffices to show that there are no primitive subspaces except $M^{0}$ w.r.t. the action of $P_{n}^{2} P_{n+1}$; this is achieved by an easy computation. In particular, we can show that the primitive monomial $Q_{n}^{\ell}$ generates the whole module $M$ : a general element $Q^{\mu}$ of $H^{0}\left(\widetilde{X}, \mathcal{O}\left(\ell D_{0}\right)\right)$ is associated to a multi-index $\mu$ such that $\sum_{i=1}^{n} \mu_{i}-2 \mu_{n+1}=\ell$. The differential operator $D=Q^{\mu} P_{n}^{\ell} / \ell$ ! belongs to $\mathcal{D}_{\mathcal{O}\left(\ell D_{0}\right)}(\widetilde{X})$ by construction, and it is easy to verify that $Q^{\mu}$ is obtained from $Q_{n}^{\ell}$ by applying $D$.

We consider now a non-positive integer $\ell$. 
Theorem 5.6. For any non-positive integer $\ell$, there is an isomorphism

$$
H^{0}\left(\widetilde{X}, \mathcal{O}\left(\ell D_{0}\right)\right) \cong \begin{cases}L_{\mathfrak{s p}_{2 n}}\left(-\frac{1}{2} \varpi_{n}\right), & \text { if } \ell \text { is even, } \\ L_{\mathfrak{s p}_{2 n}}\left(\varpi_{n-1}-\frac{3}{2} \varpi_{n}\right), & \text { if } \ell \text { is odd, }\end{cases}
$$

of $\mathfrak{g}$-modules.

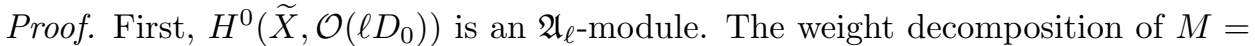
$H^{0}\left(\widetilde{X}, \mathcal{O}\left(\ell D_{0}\right)\right)$ corresponds, by the same arguments in the proof of Theorem [5.5. to the decomposition of Theorem 2.2 .

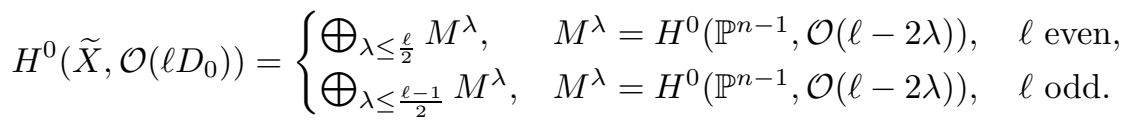

The factors in both direct sums are well known to be irreducible, finite-dimensional highest-weight modules for $\mathfrak{s l}_{n}$, which can be identified with $L_{\mathfrak{s l}_{n}}\left((\ell-2 \lambda) \varpi_{n-1}\right)$. The infinite sequence of weights w.r.t. $\mathfrak{z} \ell$ is entirely contained in the set of negative integers; hence, the requirements of Theorem 3.3 are satisfied, and $M$ is a $\mathfrak{g}$-module.

Using the results of Subsection 2.3, in particular (2), the only primitive vectors of $H^{0}\left(\widetilde{X}, \mathcal{O}\left(\ell D_{0}\right)\right)$ are (i) $Q_{n+1}^{-\frac{\ell}{2}}$, if $\ell$ is even, and (ii) $Q_{n} Q_{n+1}^{-\frac{\ell-1}{2}}$, if $\ell$ is odd. Moreover, both primitive vectors are weight vectors, with respective weights $-\frac{1}{2} \varpi_{n}$ and $\varpi_{n-1}-\frac{3}{2} \varpi_{n}$. It remains to show that they generate the respective cohomology groups as $\mathfrak{g}$-modules; in fact, to any monomial $Q^{\mu}$ in $H^{0}\left(\tilde{X}, \mathcal{O}\left(\ell D_{0}\right)\right)$, where $\mu$ is a multi-index satisfying $\sum_{i=1}^{n} \mu_{i}-2 \mu_{n+1}=\ell$, we can associate the differential monomials $Q^{\mu} P_{n+1}^{-\frac{\ell}{2}} /\left(-\frac{\ell}{2}\right)$ !, $\ell$ even, and $Q^{\mu} P_{n} P_{n+1}^{-\frac{\ell-1}{2}} /\left(-\frac{\ell-1}{2}\right)$ !, $\ell$ odd. It is easy to verify that both differential monomials are elements of $\mathcal{D}_{\mathcal{O}\left(\ell D_{0}\right)}(\tilde{X})$ in the respective cases. Hence, the 0-th cohomology is irreducible, being a highest weight module with exactly one primitive vector in both cases $\ell$ even and odd, whence the claim.

Remark 5.7. Observe that, for $\ell$ even, there is an isomorphism of $\mathfrak{g}$-modules between the module $\mathcal{O}(\bar{X})$ of regular functions on $\bar{X}$ of $\mathfrak{g}$ and the module $H^{0}\left(\widetilde{X}, \mathcal{O}\left(\ell D_{0}\right)\right)$, induced by mapping the highest weight vector 1 of $\mathcal{O}(\bar{X})$ to the highest weight vector $Q_{n+1}^{\frac{\ell}{2}}$ of $H^{0}\left(\widetilde{X}, \mathcal{O}\left(\ell D_{0}\right)\right) ; \mathcal{O}(\bar{X})$ was proved to be an irreducible highest weight $\mathfrak{g}$-module by other methods in [6]. On the other hand, the highest weight of $H^{0}\left(\widetilde{X}, \mathcal{O}\left(\ell D_{0}\right)\right)$, for $\ell$ odd, belongs to the same Weyl orbit of the highest weight of $H^{0}\left(\widetilde{X}, \mathcal{O}\left(\ell D_{0}\right)\right)$, for $\ell$ even, as can be verified by an easy computation.

As for the $n-1$-th cohomology $H^{n-1}\left(\widetilde{X}, \mathcal{O}\left(\ell D_{0}\right)\right)$, we need only consider the case $\ell \leq-n$ (otherwise it is trivial).

Theorem 5.8. For any integer $\ell \leq-n$, there is a weight space decomposition

$$
H^{n-1}\left(\tilde{X}, \mathcal{O}\left(\ell D_{0}\right)\right) \cong \bigoplus_{\left\lceil\frac{\ell+n}{2}\right\rceil \leq \lambda \leq 0} L_{\mathfrak{s I}_{n}}\left(-(\ell+n-2 \lambda) \varpi_{1}\right)
$$

in the category $\mathcal{O}_{\mathfrak{A}_{\ell}}$; the module $H^{n-1}\left(\widetilde{X}, \mathcal{O}\left(\ell D_{0}\right)\right)$ is irreducible and is of highest weight. 
Proof. We first show that $H^{n-1}\left(\widetilde{X}, \mathcal{O}\left(\ell D_{0}\right)\right)$ belongs to the category $\mathcal{O}_{\mathfrak{A}_{\ell}}$; this is a consequence of Theorem 2.2, namely

$$
M=H^{n-1}\left(\tilde{X}, \mathcal{O}\left(\ell D_{0}\right)\right)=\bigoplus_{\left\lceil\frac{\ell+n}{2}\right\rceil \leq \lambda \leq 0} M^{\lambda}, \quad M^{\lambda}=H^{n-1}\left(\mathbb{P}^{n-1}, \mathcal{O}(\ell-2 \lambda)\right) .
$$

Every weight subspace $M^{\lambda}$ is an irreducible highest weight $\mathfrak{s l}_{n}$-module with highest weight vector $Q_{1}^{\ell+n-2 \lambda-1} Q_{2}^{-1} \cdots Q_{n}^{-1} Q_{n+1}^{-\lambda}$ and corresponding highest weight $-(\ell+n-2 \lambda) \varpi_{1}$, whence the identification $M^{\lambda}=L_{\mathfrak{s l}_{n}}\left(-(\ell+n-2 \lambda) \varpi_{1}\right)$; observe that the highest weight vector is a multiple of $e_{-\beta}^{-\lambda}\left(Q_{1}^{\ell+n-1} Q_{2}^{-1} \cdots Q_{n}^{-1}\right), \beta$ being the highest root of $\mathfrak{g}$. Finally, $M$ is generated by the highest weight vector of $M^{0}$

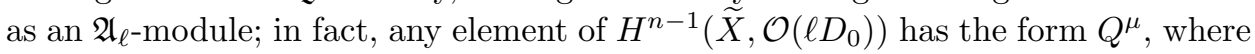
$\mu_{i}<0, i=1, \ldots, n, \mu_{n+1} \geq 0$, and $\sum_{i=1}^{n} \mu_{i}-2 \mu_{n+1}=\ell$. To such a monomial, we associate the differential operator

$$
D_{\mu}=\frac{(-1)^{\sum_{i=1}^{n} \mu_{i}+n}}{\prod_{i=0}^{-\mu_{1}}(\ell+n-1-i) \prod_{j=2}^{n}\left(-\left(\mu_{j}+1\right)\right) !} Q_{1}^{-\ell-n} Q_{n+1}^{\mu_{n+1}} P_{1}^{-\mu_{1}-1} \cdots P_{n}^{-\left(\mu_{n}+1\right)}
$$

which is readily checked to belong to $\mathcal{D}_{\mathcal{O}\left(\ell D_{0}\right)}(\tilde{X})$; it is also easy to verify that $Q^{\mu}=D_{\mu}\left(Q_{1}^{\ell+n-1} Q_{2}^{-1} \cdots Q_{n}^{-1}\right)$ modulo coboundaries.

5.2.2. The module structure on the cohomology of $Y$. For any even integer $\ell$, we consider the cohomology of the weighted $n$-dimensional projective space $Y$ with values in the Serre bundle $\mathcal{O}(\ell)$, see Subsection 4.2. As a byproduct of Corollary [5.3, the cohomology groups $H^{\bullet}(X, \mathcal{O}(\ell))$ inherit the structure of $\mathfrak{A}$-modules. Recall the results of Subsection 4.2 concerning the cohomology of $Y$ with values in $\mathcal{O}(\ell)$. Recalling Theorem [5.1, the Fourier transform $F_{I}$, for $I$ as in Subsection 5.1, is used to compute the image of $\mathfrak{A}_{\ell+2}$ in $\mathcal{D}_{\mathcal{O}(\ell)}(Y)$ from $\mathcal{D}_{\mathcal{O}\left((\ell+2) D_{0}\right)}(\tilde{X})$. Since the $I$-reflection affects only the generating vector $k_{n+1}^{\prime}$, the only generators of $\mathcal{D}_{\mathcal{O}\left((\ell+2) D_{0}\right)}(\widetilde{X})$ affected by $F_{I}$ are

$$
\begin{aligned}
F_{I}\left(\frac{1}{2} P_{n}^{2} P_{n+1}\right) & =-\frac{1}{2} P_{n}^{2} Q_{n+1}, \\
F_{I}\left(-Q_{n+1} P_{n+1}\right) & =Q_{n+1} P_{n+1}+1 .
\end{aligned}
$$

Similarly to what was done in Subsubsection 5.2.1, we can identify the cohomology of $X$ with values in $\mathcal{O}(\ell)$, for any even integer $\ell$, with certain irreducible, highest weight $\mathfrak{A}_{\ell+2}$-modules in the category $\mathcal{O}_{\mathfrak{A}_{\ell+2}}$.

Theorem 5.9. For any even, non-negative integer $\ell$, there is a weight space decomposition

$$
H^{0}(Y, \mathcal{O}(\ell)) \cong \bigoplus_{1 \leq \lambda \leq \frac{\ell+2}{2}} L_{\mathfrak{s l}_{n}}\left((\ell-2 \lambda+2) \varpi_{n-1}\right)
$$

in the category $\mathcal{O}_{\mathfrak{A}_{\ell+2}}$; the module $H^{0}(Y, \mathcal{O}(\ell))$ is irreducible and of highest weight.

Proof. The global sections of $\mathcal{O}(\ell)$ are spanned by monomials $Q^{\mu}$, with multi-indices $\mu$ satisfying $\sum_{i=1}^{n} \mu_{i}+2 \mu_{n+1}=\ell$; hence, $0 \leq \mu_{n+1} \leq \frac{\ell}{2}$. The weight subspaces $M^{\lambda}$ of $M=H^{0}(Y, \mathcal{O}(\ell))$ correspond to weights $1 \leq \lambda \leq \frac{\ell+2}{2}$; in fact, $M$ splits as $M=\bigoplus_{1 \leq m \leq \frac{\ell+2}{2}} M^{\lambda}$, with $M^{\lambda}$ the space of complex polynomials in $n$ variables, homogeneous of degree $\ell-2 \lambda+2$, the isomorphism being concretely

$$
M^{\lambda} \ni Q_{1}^{\mu_{1}} \cdots Q_{n}^{\mu_{n}} \mapsto Q_{1}^{\mu_{1}} \cdots Q_{n}^{\mu_{n}} Q_{n+1}^{\lambda-1} \in M .
$$


Every weight subspace $M^{\lambda}$ is an irreducible, finite-dimensional highest weight module for $\mathfrak{s l}_{n}$. The highest weight vector is $Q_{n}^{\ell-2 \lambda+2} Q_{n+1}^{\lambda-1}$ with highest weight $(\ell-2 \lambda+2) \varpi_{n-1}$, whence the identification $L_{\mathfrak{s t}_{n}}\left((\ell-2 \lambda+2) \varpi_{n-1}\right)$. Observe that the $\mathfrak{s l}_{n}$-highest weight vector of $M^{\lambda}$ is a multiple of $e_{-\alpha_{n}}^{\ell-2 \lambda+2}\left(Q_{n+1}^{\frac{\ell}{2}}\right), 0 \leq \lambda \leq \frac{\ell}{2}$, with $Q_{n+1}^{\frac{\ell}{2}}$ the highest weight vector of $M^{\frac{\ell+2}{2}}$. Therefore, $H^{0}(Y, \mathcal{O}(\ell))$ belongs to the category $\mathcal{O}_{\mathfrak{A}_{\ell+2}}$; its highest weight w.r.t. $\mathfrak{z} \ell+2$ belongs to $\mathbb{N}$, the sequence of corresponding weights has finitely many terms, hence Theorem 3.3 does not apply.

There is only one primitive one-dimensional subspace, spanned by the vector $Q_{n+1}^{\frac{\ell}{2}}$ in $H^{0}(Y, \mathcal{O}(\ell))$; hence, the module is irreducible. The generator $Q_{n+1}^{\frac{\ell}{2}}$ is a highest weight vector, which additionally generates $H^{0}(Y, \mathcal{O}(\ell))$ as an $\mathcal{O}_{\mathfrak{A}_{\ell+2}}{ }^{-}$ module by computations similar to those in the proof of Theorem 5.6

As for the $n$-th cohomology of $Y$ with values in $\mathcal{O}(\ell)$, for an even integer $\ell$, we have

Theorem 5.10. If $\ell$ is any even integer, such that $\ell \leq-n-2$, there is a weight space decomposition

$$
H^{n}(Y, \mathcal{O}(\ell)) \cong \bigoplus_{\left\lceil\frac{\ell+n}{n}\right\rceil \leq \lambda \leq 0} L_{\mathfrak{s} I_{n}}\left(-(\ell+n-2 \lambda+2) \varpi_{1}\right)
$$

of $\mathfrak{A}_{\ell+2}$-modules; furthermore, $H^{n}(Y, \mathcal{O}(\ell))$ is irreducible and is generated by a highest weight vector.

Proof. We recall that $M=H^{n}(Y, \mathcal{O}(\ell))$ is spanned by negative monomials $Q^{\mu}$, with $\mu_{i}<0, i=1, \ldots, n+1$, and $\sum_{i=1}^{n} \mu_{i}+2 \mu_{n+1}=\ell$, thus, we have the splitting

$$
M=\bigoplus_{\left\lceil\frac{\ell+n}{2}\right\rceil \leq m \leq-1} S_{m}^{n}
$$

where $S_{m}^{n}$ denotes the subspace of negative monomials in $n$ variables of the form $Q_{1}^{\mu_{1}} \cdots Q_{n}^{\mu_{n}}$ with $\mu_{i}<0$ and $\sum_{i=1}^{n} \mu_{i}=\ell-2 m$, the isomorphism being given by

$$
S_{m}^{n} \ni Q^{\mu_{1}} \cdots Q_{n}^{\mu_{n}} \mapsto Q_{1}^{\mu_{1}} \cdots Q_{n}^{\mu_{n}} Q_{n+1}^{m} \in M .
$$

(Observe that the negative monomials must be considered modulo coboundaries.) The subspace $S_{m}^{n}$ is an irreducible highest weight module for $\mathfrak{s l}_{n}$; the highest weight vector is $Q_{1}^{\ell+n-2 m-1} Q_{2}^{-1} \cdots Q_{n}^{-1}$ with highest weight $-(\ell+n-2 m) \varpi_{1}$, hence, recalling the $\mathfrak{z}_{\ell+2}$-action on $M, M^{\lambda}=L_{\mathfrak{s l}_{n}}\left(-(\ell+n-2 \lambda+2) \varpi_{1}\right)$ module $M$ belongs to the category $\mathcal{O}_{\mathfrak{A}_{\ell+2}}$; by the previous arguments it is finite-dimensional; the only primitive subspace of $M$ corresponds, by direct computations, to $M^{0}$, whence $M$ is irreducible. Finally, the highest weight vector of $M^{0}$ generates $M$ as an $\mathfrak{A}_{\ell+2^{-}}$ module by slight modifications of the arguments in the proof of Theorem 5.8

\section{ACKNOWLEDGMENTS}

The author thanks Giovanni Felder for many useful discussions and many helpful comments and Thierry Levasseur for having carefully read the first draft of this manuscript and suggesting better terminology; the author is also greatly indebted to the referee of Representation Theory for many useful comments and for having pointed out a mistake and some gaps in the proof of the results of Subsection 2.3. 


\section{REFERENCES}

[1] Walter Borho and Jean-Luc Brylinski, Differential operators on homogeneous spaces. I. Irreducibility of the associated variety for annihilators of induced modules, Invent. Math. 69 (1982), no. 3, 437-476. MR679767 (84b:17007)

[2] Nicolas Bourbaki, Lie groups and Lie algebras. Chapters 4-6, Elements of Mathematics (Berlin), Springer-Verlag, Berlin, 2002. Translated from the 1968 French original by Andrew Pressley. MR.1890629 (2003a:17001)

[3] Giovanni Felder and Carlo A. Rossi, Differential operators on toric varieties and Fourier transform (2007), available at http://arxiv.org/abs/math/0705.1709v3

[4] William Fulton, Introduction to toric varieties, Annals of Mathematics Studies, vol. 131, Princeton University Press, Princeton, NJ, 1993. The William H. Roever Lectures in Geometry. MR.1234037 (94g:14028)

[5] Robin Hartshorne, Algebraic geometry, Springer-Verlag, New York, 1977. Graduate Texts in Mathematics, No. 52. MR0463157 (57:3116)

[6] T. Levasseur, S. P. Smith, and J. T. Stafford, The minimal nilpotent orbit, the Joseph ideal, and differential operators, J. Algebra 116 (1988), no. 2, 480-501. MR953165 (89k:17028)

[7] Ian M. Musson, Actions of tori on Weyl algebras, Comm. Algebra 16 (1988), no. 1, 139-148. MR.921946 (88k:17013)

[8] , Differential operators on toric varieties, J. Pure Appl. Algebra 95 (1994), no. 3, 303-315. MR1295963(95i:16026)

[9] Ian M. Musson and Sonia L. Rueda, Finite dimensional representations of invariant differential operators, Trans. Amer. Math. Soc. 357 (2005), no. 7, 2739-2752 (electronic). MR2139525 (2006a:16034)

[10] Michel Van den Bergh, Differential operators on semi-invariants for tori and weighted projective spaces, Topics in invariant theory (Paris, 1989/1990), Lecture Notes in Math., vol. 1478, Springer, Berlin, 1991, pp. 255-272. MR1180993 (93h:16046)

Department of mathematics, ETH Zürich, 8092 Zürich, Switzerland

E-mail address: crossi@math.ethz.ch 